\title{
Transgenic mice expressing an inhibitory truncated form of p300 exhibit long-term memory deficits
}

\author{
Ana M.M. Oliveira, ${ }^{1,2}$ Marcelo A. Wood, ${ }^{3}$ Conor B. McDonough, ${ }^{1}$ and Ted Abel ${ }^{1,4}$ \\ ${ }^{1}$ Department of Biology, University of Pennsylvania, Philadelphia, Pennsylvania 19104, USA; ${ }^{2}$ Gulbenkian Ph.D. Programme in \\ Biomedicine, Instituto Gulbenkian de Ciência, 2781-901 Oeiras, Portugal; ${ }^{3}$ Department of Neurobiology and Behavior, Center for \\ the Neurobiology of Learning and Memory, University of California, Irvine, California 92697, USA
}

\begin{abstract}
The formation of many forms of long-term memory requires several molecular mechanisms including regulation of gene expression. The mechanisms directing transcription require not only activation of individual transcription factors but also recruitment of transcriptional coactivators. CBP and p300 are transcriptional coactivators that interact with a large number of transcription factors and regulate transcription through multiple mechanisms, including an intrinsic histone acetyltransferase (HAT) activity. HAT activity mediates acetylation of lysine residues on the amino-terminal tails of histone proteins, thereby increasing DNA accessibility for transcription factors to activate gene expression. CBP has been shown to play an important role in long-term memory formation. We have investigated whether p300 is also required for certain forms of memory. p300 shares a high degree of homology with CBP and has been shown to interact with transcription factors known to be critical for long-term memory formation. Here we demonstrate that conditional transgenic mice expressing an inhibitory truncated form of p300 (p300 1 ), which lacks the carboxy-terminal HAT and activation domains, have impaired long-term recognition memory and contextual fear memory. Thus, our study demonstrates that p300 is required for certain forms of memory and that the HAT and carboxy-terminal domains play a critical role.
\end{abstract}

Information is first stored as a short-term memory lasting minutes to hours and can then be stabilized into long-term memory lasting days to lifetime. These forms of memory differ in that the formation of long-term memory requires activation of transcription (for review, see Korzus 2003). Transcriptional activation requires recruitment of a large number of proteins in addition to individual transcription factors. Cyclic AMP-responsive element binding protein (CREB) binding protein (CBP) and its homolog E1A binding protein (p300) are transcriptional coactivators (Chrivia et al. 1993; Eckner et al. 1994) that interact with multiple transcriptional factors to facilitate gene-specific transcription (for review, see Vo and Goodman 2001).

Several studies have shown that CBP plays an important role in long-term memory formation (Oike et al. 1999; Bourtchouladze et al. 2003; Alarcon et al. 2004; Korzus et al. 2004; Wood et al. 2005, 2006). The first demonstration that CBP may play a role in memory formation came from a study in which genetically modified mice exhibited long-term memory deficits as well as developmental defects that resembled the phenotype observed in Rubinstein Taybi syndrome (RTS) patients. In these mice, a single $\mathrm{Cbp}$ allele is truncated (truncated protein contains residues 1-1084), and this truncated form is expressed throughout developmental and adult stages (Oike et al. 1999). In our laboratory, transgenic mice that express the same truncation form (CBP $\Delta 1)$ only in adulthood and in forebrain neurons were generated to study the role of CBP in memory independently of its role in development (Wood et al. 2005). CBP $\Delta 1$ transgenic mice exhibit deficits in specific forms of hippocampal synaptic plasticity and long-term memory formation. In the present study we have investigated whether p300 is also required for long-term memory formation.

Recently, a screen for mutations in RTS patients showed that only $40 \%$ of the patients carried mutations in the $C B P$ gene,

\footnotetext{
${ }^{4}$ Corresponding author.
}

E-mail abele@sas.upenn.edu; fax (215) 898-8780.

Article is online at http://www.learnmem.org/cgi/doi/10.1101//m.656907. suggesting that mutations in other genes could also be the cause of this syndrome (Roelfsema et al. 2005). A potential candidate is the EP300 gene, encoding the coactivator p300, because of its high degree of homology with $C B P$. Indeed, some RTS patients carry mutations in the $E P 300$ gene that lead to proteins that do not contain the HAT domain (Roelfsema et al. 2005). Although all RTS patients have varying degrees of cognitive impairment and mental retardation, the phenotypes of patients with mutations in either the CBP or EP300 genes do not overlap completely; RTS patients with mutations in the EP300 do not have the skeletal abnormalities that are usually observed in patients with mutations in the CBP gene (Bartholdi et al. 2007). The phenotypic differences between RTS patients with mutations in the $C B P$ gene and EP300 gene and the observation that CBP and p300 have different functions during embryogenesis and hematopoiesis (Tanaka et al. 1997; Yao et al. 1998; Kasper et al. 2002, 2006; for review, see also Kalkhoven 2004) suggest that CBP and p300, despite their high degree of homology, also have unique functions in vivo. In support of this idea, we have recently found that $\mathrm{CBP}$ and $\mathrm{p} 300$ have distinct roles in motor skill learning (Oliveira et al. 2006).

CBP and p300 regulate transcription through multiple mechanisms. CBP and p300 function as scaffolds that form macromolecular regulatory complexes, linking gene-specific transcription factors to the basal transcription machinery. Furthermore, CBP and p300 contain intrinsic histone acetyltransferase (HAT) activity in the carboxy-terminal domain that mediates acetylation of lysine residues on the amino-terminal tails of histone proteins (for review, see Chan and La Thangue 2001). Acetylation neutralizes the positively charged lysine residues in histones and disrupts the interaction between histones and DNA, increasing DNA accessibility for transcription factors to activate gene expression (Grunstein 1997). In addition to HAT activity, $\mathrm{CBP}$ and $\mathrm{p} 300$ have also been shown to acetylate other proteins, including transcription factors ( $\mathrm{Gu}$ and Roeder 1997; Furia et al. 2002). p300 HAT activity has been shown to be highest in the adult mouse brain compared with other tissues, suggesting that 
this protein may play an important role in the regulation of gene transcription in the brain ( $\mathrm{Li}$ et al. 2002). p300 has also been shown to interact in vitro with transcription factors that are known to play a role in learning and memory, such as CREB, NF-кB, Elk-1, and C/EBPß (Lundblad et al. 1995; Li et al. 2003; Romano et al. 2006; Cesena et al. 2007).

Thus, p300 functions in vitro and cell culture as a transcriptional coactivator for transcription factors known to be critical in memory. p300 is highly homologous to CBP, and mutations in CBP produce memory deficits in mice. Mutations in $C B P$ or EP300 produce similar cognitive impairments in RTS patients. Therefore, we have directly investigated whether p300 plays a role in long-term memory formation using genetically modified mice expressing a truncated form of p300 (p300 1 ). p300 1 lacks the carboxy-terminal portion of the protein and therefore does not contain the HAT domain. Transgenic mice were generated using the tetracycline system to allow for temporal and regional control of the expression of the transgene. We show that expression of p300 1 is associated with deficits in long-term recognition memory and contextual fear memory, suggesting that p300 is involved in these forms of memory.

\section{Results}

\section{p300 expression in the mouse brain}

We first examined the expression of p300 in the mouse brain as this has not been established. We used antibodies shown to be specific for p300 in previous studies (Kasper et al. 2006). Kasper et al. (2006) used the same antibodies to show by immunohistochemistry that p300 is absent in T-cells derived from p300 conditional knockout mice. We show that p300 is expressed ubiquitously in the mouse brain, including areas that are critical for long-term memory formation such as hippocampus, amygdala, and cortex (Fig. 1).

\section{Generation and characterization of p300 $\Delta 1$ transgenic mice}

Many lines of evidence suggest that CBP plays a critical role in the formation of certain types of long-term memory (Oike et al.
A

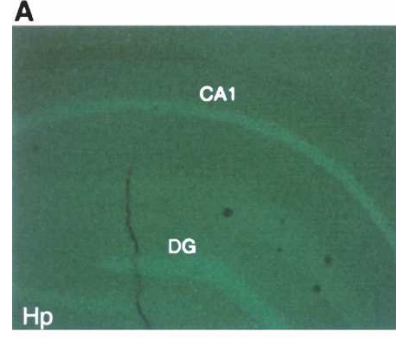

c

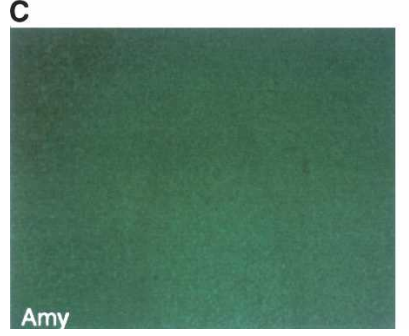

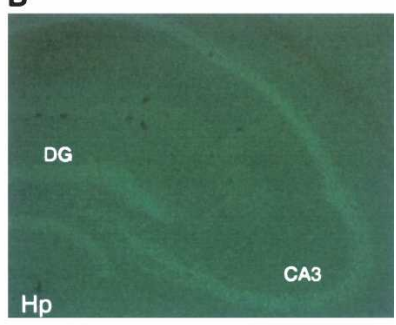

D

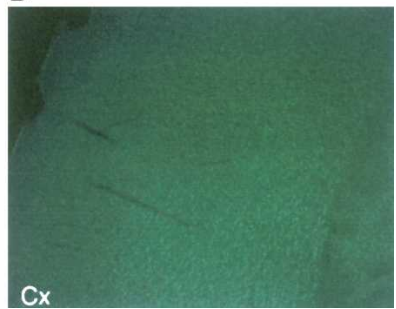

Figure 1. p300 expression in the brain. Fluorescent immunohistochemistry of p300 in brain coronal sections from naïve C57BI/6J mice. p300 is ubiquitously expressed in the mouse forebrain including the CA1, $\mathrm{CA} 3$, and DG regions of the hippocampus $(\mathrm{Hp})(A, B)$, amygdala (Amy) $(C)$, and cortex $(C x)(D)$.
1999; Bourtchouladze et al. 2003; Alarcon et al. 2004; Korzus et al. 2004; Wood et al. 2005, 2006). It is not known, however, whether its homolog p300 is also required for long-term memory formation. To address this question, we generated transgenic mice expressing a truncated form of p300 (p300 1 ) that lacks the carboxy-terminal part of p300 (Fig. 2A). We chose a conditional transgenic system in which expression of the transgene could be spatially and temporally restricted.

To spatially restrict the expression of the transgene to forebrain neurons, we used one transgenic mouse line (TG1 in Fig. 2C) that contains the tetracycline responsive transactivator (tTA) under the control of the CaMKII $\alpha$ promoter (CaMKII $\alpha$-tTA line B) (Mayford et al. 1996). To temporally restrict the expression of the transgene, TG1 was combined with a second transgenic mouse line (TG2 in Fig. 2C) that contains the p300 1 transgene driven by the tetracycline operator sequence (tetO) and a minimal promoter (Mayford et al. 1996) (tetOp30041). Crossing both transgenic lines generates bitransgenic mice (referred to in this paper as p $300 \Delta 1$ transgenic mice) in which expression of the transgene should be restricted to excitatory neurons in the adult mouse forebrain and suppressed by administration of doxycycline (dox) in the animals' food (Fig. 2C). We generated three lines of tetOp $300 \Delta 1$ transgenic mice and the line with higher copy number (determined by Southern blot, data not shown) was used in the present study as this line showed the strongest impairment in contextual fear conditioning in an initial behavioral screening of all three lines.

CREB is one of the many transcription factors that interacts with p300 through the kinase inducible interaction domain (KIX) (Vo and Goodman 2001), and it has been shown to require the HAT activity of p300 for transcriptional activation (Lundblad et al. 1995; Yuan and Gambee 2001). Therefore, we predicted that p300 1 expression would impair CRE-dependent transcription. We observed that CRE-dependent transcription induced by forskolin and 3-isobutyl-1-methylxanthine (IBMX) is significantly inhibited $\left(t_{(5)}=4.95, P<0.05\right)$ when HEK293 cells are cotransfected with p300 $\Delta 1$ and a CRE-luciferase reporter (Fig. 2B). p300 1 is likely acting in an inhibitory fashion by competing with endogenous p300 for p300-specific binding sites, thus compromising the function of proteins, such as CREB, that interact with the amino-terminal part of p300 and require its HAT and/or other carboxy-terminal domain.

p300 1 transgenic mice do not show gross morphological abnormalities or reduced size compared with wild-type or single transgenic littermates. Similarly, p300 1 transgenic mice on dox appear normal (data not shown), suggesting that p300 1 transgenic mice are developmentally normal. To assess the expression of the transgene, we performed reverse transcriptase-polymerase chain reaction (RT-PCR) analysis of cDNA prepared from RNA from amygdala, hippocampus, cortex, and cerebellum of p300 1 transgenic and wild-type mice (Fig. 2D). We chose PCR primers that span an intron to discriminate between transgene genomic DNA and transgene cDNA. We observed the presence of the p300 1 transgene after PCR analysis of cDNA from all brain regions analyzed in p300 1 transgenic mice both on and off dox (Fig. 2D). The identity of the bands was confirmed by restriction enzyme analysis. These observations suggest that transgene expression is not restricted to the forebrain and is not suppressed by dox treatment. However, because this approach is not quantitative, we could not determine whether dox regulation is totally absent or just incomplete. Therefore, we examined how transgene expression affects acetylation of histone $\mathrm{H} 3$ in the forebrain of p300 1 transgenic mice and their wild-type littermates (Fig. 2E) by Western blot. We chose to measure acetylation of the lysines $\mathrm{K} 9$ and $\mathrm{K} 14$ in histone $\mathrm{H} 3$ because it has been previously shown to be enhanced in the hippocampus after contextual fear 
A

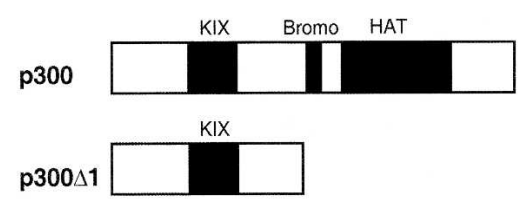

C

Transgene expression on

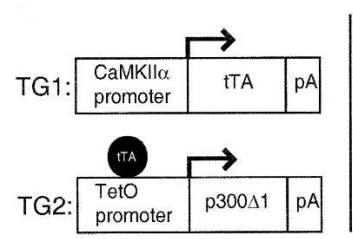

Transgene expression off

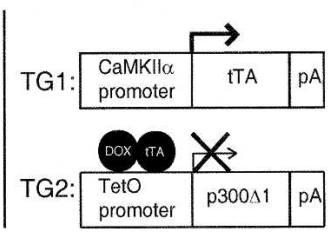

B

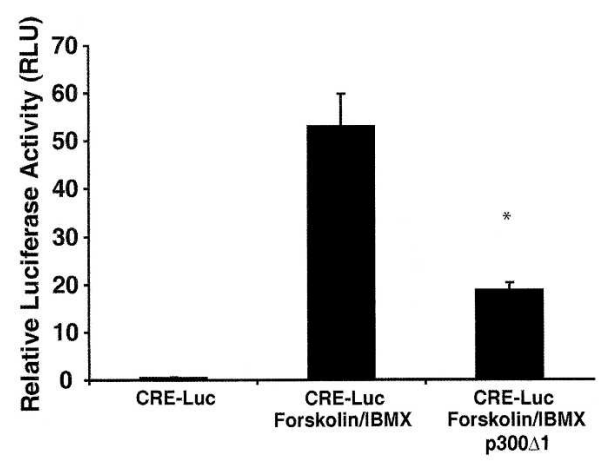

D

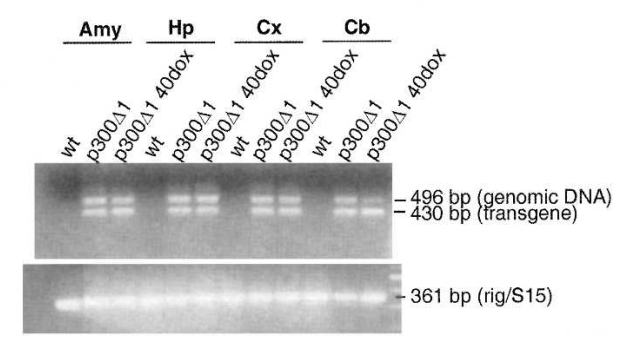

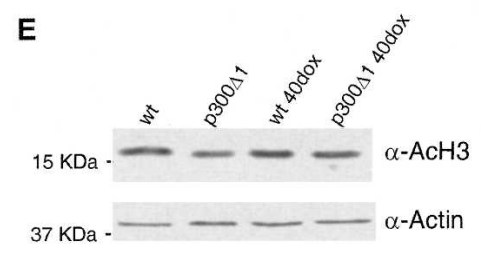

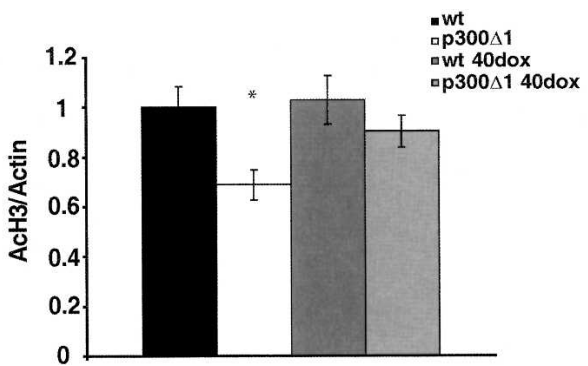

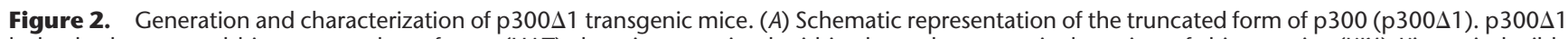
lacks the bromo and histone acetyltransferase (HAT) domains contained within the carboxy-terminal portion of this protein. (KIX) Kinase inducible

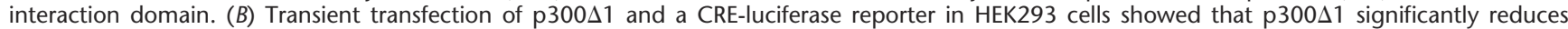
CRE-dependent transcription induced by forskolin and IBMX. (C) Schematic representation of the tetracycline system used to regionally and temporally

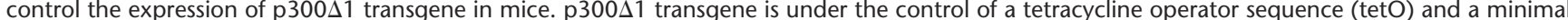
promoter and mice carrying this transgene were generated and crossed to mice that contain the tTA transactivator driven by the CaMKIl $\alpha$ promoter (line B, Mayford et al. 1996) giving rise to bitransgenic mice (referred to in this paper as p300 1 transgenic mice). In the absence of doxycycline (dox),

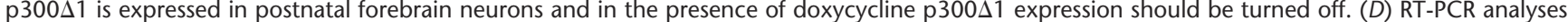
of cDNA synthesized from RNA isolated from amygdala (Amy), hippocampus (Hp), cortex (Cx), and cerebellum (Cb) of wild-type and p300 1 transgenic mice on and off dox. (E) Western blot analysis of levels of acetylated lysines K9 and K14 in histone H3 (AcH3) in forebrain of wild-type (wt)

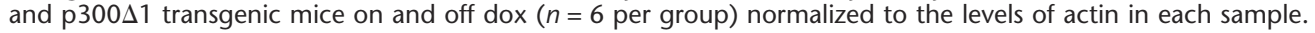

conditioning (Levenson et al. 2004). Furthermore, lysines K9 and K14 in histone H3 are p300 targets (McManus and Hendzel 2003). We observed a significant effect of genotype $\left(F_{(1,20)}=7.94\right.$, $P<0.05)$. A direct comparison of the groups showed that $\mathrm{p} 300 \Delta 1$ transgenic mice off dox have significantly lower levels of acetylated histone $\mathrm{H} 3$ (AcH3) in the forebrain than their wild-type littermates $(P<0.05)$. Wild-type animals on dox show similar levels of AcH3 as compared with wild-type animals off dox, showing that dox does not affect histone $\mathrm{H} 3$ acetylation. p300 1 transgenic mice on dox show slightly reduced levels of AcH3 compared with wild-type mice, but this reduction was not statistically significant $(P=0.38)$. This finding suggests that $\mathrm{p} 300 \Delta 1$ transgenic mice on dox still express the transgene in the forebrain but at lower levels than in p300 1 transgenic mice off dox 
and that these levels are not enough to produce a significant reduction of acetylation of lysines K9 and K14 in histone H3.

The presence of the transgene in the cerebellum (Fig. 2D) is perhaps not surprising because CaMKII $\alpha$ is expressed in Purkinje cells of the cerebellum; however, the level of CaMKII $\alpha$ in the cerebellum is much lower overall than in the forebrain (Burgin et al. 1990; Hanson and Schulman 1992; Zou et al. 2002). We observed that the levels of AcH3 in the cerebellum of wild-type and p300 1 transgenic mice are not different (data not shown). However, it remains possible that histone acetylation is reduced in Purkinje cells, but, since the levels of CaMKII $\alpha$ in these cells are very low and the majority of cells in the cerebellum are granule cells, we do not observe a significant difference when analyzing the levels of AcH3 in the whole cerebellum of p300 1 transgenic mice when compared with wild-type littermates by Western blot. Also, p300 1 transgenic mice show normal locomotor activity (see below) despite expression of the transgene in the cerebellum.

Overall, p300 1 transgene expression seems to be incompletely regulated by dox and not fully restricted to the forebrain, but the levels of leaky expression in the presence of dox are not sufficient to cause a significant effect on acetylation of lysines $\mathrm{K} 9$ and $\mathrm{K} 14$ of histone H3.

\section{p300 1 transgenic mice show normal spatial memory} but impaired recognition memory

To assess spatial memory, p300 1 transgenic mice and wild-type littermates were tested in the hidden platform version of the Morris water maze task (Fig. 3). The ability of rodents to acquire this task has been shown to depend on an intact hippocampus (Morris et al. 1982). p30041 transgenic mice and wild-type littermates showed a significant improvement across the acquisition phase (effect of training day $F_{(7,98)}=6.1, P<0.0001$ ), showing that both groups acquired this task. However, no effect of genotype $\left(F_{(1,98)}=2.3, P=\mathrm{NS}\right)$ or genotype $\times$ day interaction $\left(F_{(7,98)}=1.6, P=\mathrm{NS}\right)$ was observed. Overall, p300 1 transgenic mice exhibited similar latencies to find the hidden platform as their wild-type littermates (Fig. 3A). To assess spatial memory, two probe trials were performed: the first, one day after session four, and the second, one day after session eight. No differences were observed between p $300 \Delta 1$ transgenic mice and wild-type littermates in the time spent in target quadrant or number of crossings over the area where the platform was placed during acquisition in either probe trail (data not shown; Fig. 3B,C). Swim speed, thigmotaxis, and total swimming distance were also not different between genotypes, showing that p300 1 transgenic mice have normal locomotor activity despite expression of the transgene in the cerebellum. Transgenic mice also showed normal motor coordination and motor skill learning when tested in the rotarod task (data not shown).

Several studies have demonstrated a role for CBP in recognition memory (Bourtchouladze et al. 2003; Alarcon et al. 2004; Korzus et al. 2004; Wood et al. 2006). In agreement with these studies, we show here that CBP $\Delta 1$ transgenic mice generated in our laboratory (Wood et al. 2005) have a significant deficit in long-term memory for novel object recognition (Fig. 4A). We observed that CBP 1 transgenic mice had a significantly lower preference for the novel object when tested $24 \mathrm{~h}$ after training $\left(t_{(14)}=2.34, P<0.05\right)$. In contrast, when tested $30 \mathrm{~min}$ after training, the preference for the novel object was similar between CBP $\Delta 1$ transgenic and wild-type animals (Fig. 4A). This observation further confirms that CBP is important for long-term recognition memory. We then determined if $\mathrm{p} 300$ also plays a role in this type of memory (Fig. 4B,C). We observed that p $300 \Delta 1$ transgenic mice show significantly lower preference for the novel object when tested $24 \mathrm{~h}$ after training, as compared with wild-type

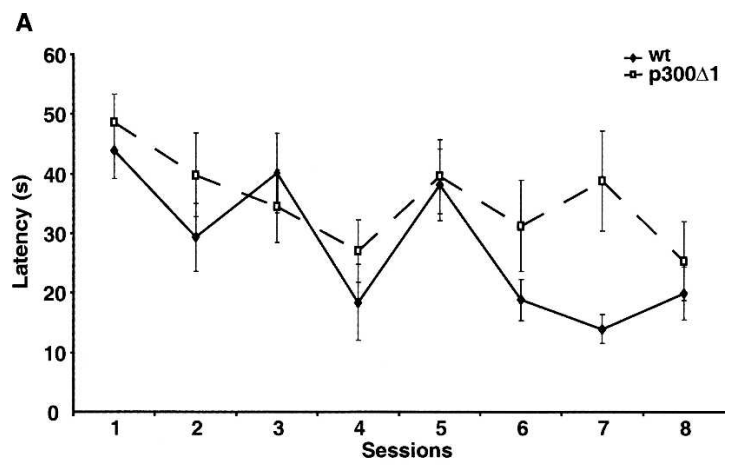

B
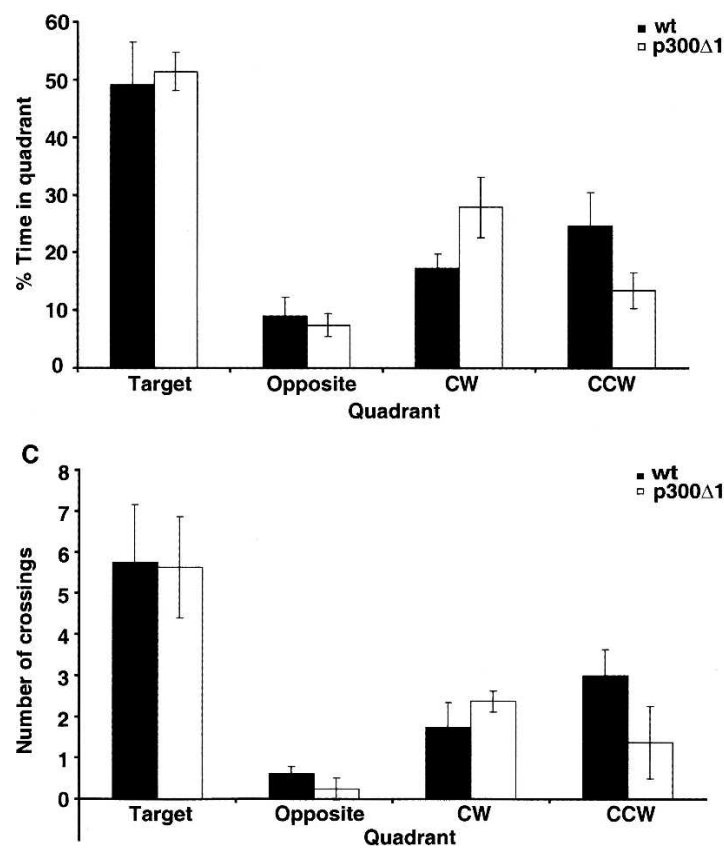

Figure 3. p300 11 transgenic mice show normal spatial memory in the Morris water maze. $(A)$ Wild-type $(n=8)$ and p300 1 transgenic $(n=8)$ mice do not show a significant difference in the latency to find the hidden platform during the acquisition phase of the task. $(B)$ During probe trial (performed after session 8), p300 1 transgenic mice and wild-type littermates showed a similar percentage of time spent swimming in the target quadrant. (C) During the probe trial (performed after session 8) the number of crossings over the area where the platform was located during acquisition was not different between wild-type and p300 1 transgenic mice.

littermates $\left(t_{(28)}=2.20, P<0.05\right)$ (Fig. 4B). Short-term recognition memory, tested 30 min after training, was normal in p $300 \Delta 1$ transgenic mice (Fig. 4B), showing that p $300 \Delta 1$ is disrupting consolidation of the learning experience without affecting acquisition of the task. The long-term recognition memory phenotype was reversed in a different set of animals that were kept on dox food from conception (Fig. 4C), indicating that dox presumably reduced the expression of p $300 \Delta 1$ transgene to levels that were not sufficient to cause an impairment. The observation that p300s 1 transgenic mice on dox do not show a significant impairment supports the idea that the phenotype of p300 $\Delta 1$ transgenic mice off dox is not due to a transgene insertion effect. The total time spent exploring both objects during training and testing phases among the different conditions tested was not different between the two genotypes (data not shown). Together, these results suggest that p300 plays a role in the formation of longterm recognition memory but not spatial memory. 
A

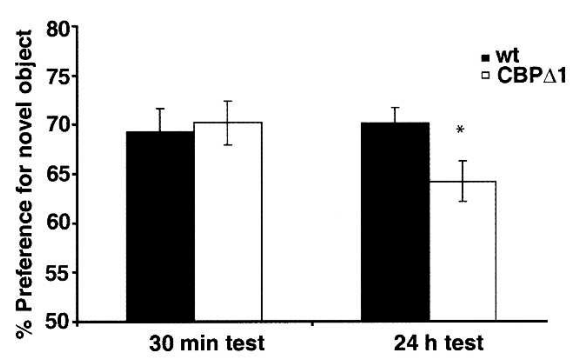

C

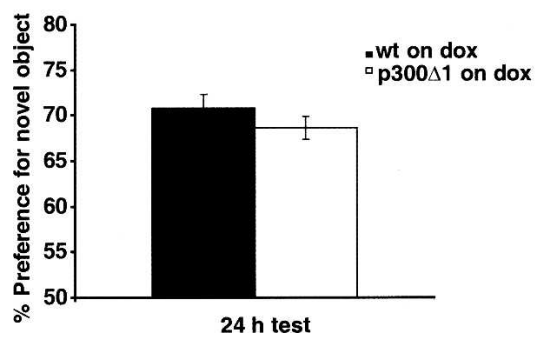

Figure 4. $C B P \Delta 1$ and $p 300 \Delta 1$ transgenic mice show impaired long-term recognition memory. $(A)$ $\mathrm{CBP} \Delta 1$ transgenic mice $(n=5)$ and wild-type littermates $(n=6)$ show similar preference for the novel object when tested 30 min after training. However, when tested $24 \mathrm{~h}$ after training, CBP $\Delta 1$ transgenic mice $(n=9)$ show significant lower preference for the novel object than their wild-type littermates

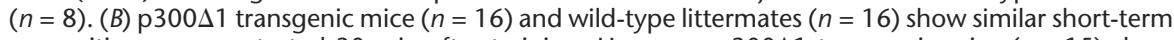
recognition memory tested 30 min after training. However, p300 1 transgenic mice $(n=15)$ show impaired novel object recognition tested $24 \mathrm{~h}$ after training compared with their wild-type littermates

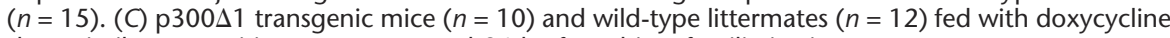
show similar recognition memory tested $24 \mathrm{~h}$ after object familiarization.

\section{p300 $\Delta 1$ transgenic mice show impaired contextual fear memory but normal cued fear memory}

We assessed the role of p300 in associative memory by using contextual and cued fear conditioning (Fig. 5). Contextual and cued fear conditioning partially differ in the neural systems required. Lesions in the hippocampus only affect contextual fear, but both tasks are sensitive to lesions in the amygdala (for review, see Maren and Quirk 2004). p300 1 transgenic mice showed significantly lower freezing compared with their wild-type littermates $\left(t_{(35)}=2.12, P<0.05\right)$ when tested $24 \mathrm{~h}$ after conditioning (Fig. 5A). In the case of mice treated with dox, we still observed a small impairment in the p300 1 transgenic mice, but this decrease was not statistically significant (Fig. 5B). This pattern is consistent with the levels of histone $\mathrm{H} 3$ acetylation in the forebrain of p300 1 transgenic and wild-type mice on and off dox (Fig. 2E). We also found that p300 $\Delta 1$ transgenic mice had normal cued fear memory at $24 \mathrm{~h}$ (Fig. 5D), normal short-term contextual fear memory at $1 \mathrm{~h}$ (Fig. 5C), and normal freezing during the $30 \mathrm{sec}$ immediately after shock. Together, these results suggest that p300 is required for consolidation of contextual fear memory but is not necessary for acquisition of contextual fear memory or for long-term cued fear memory.

\section{Discussion}

This study is the first demonstration that the transcriptional coactivator p300 plays a role in long-term memory formation. We have investigated the role of p300 in memory using genetically modified mice that express a truncated form of p300. p300 1 transgenic mice show deficits in long-term recognition memory and long-term memory for contextual fear conditioning, with no deficits in short-term memory, hippocampus-independent cued fear conditioning, or spatial learning in the Morris water maze. These results suggest that p300 is required during learning-induced transcription activation that is critical for the consolidation of memory (for review, see Korzus 2003).

We generated transgenic mice that express a truncated form of p300 (p300s1) under the control of the tetracycline system. We observed that treating p300 1 transgenic mice with dox did not completely suppress expression of the transgene. Previous studies have also shown incomplete suppression of expression of the transgene in a doxycycline-regulated system (Tremblay et al. 1998). Despite incomplete suppression of the transgene expression, p300 1 transgenic mice on dox did not show significant behavioral impairments, and extracts from p300 1 transgenic mice on dox had similar levels of acetylation of lysines $\mathrm{K} 9$ and $\mathrm{K} 14$ in histone $\mathrm{H} 3$ as extracts from wild-type littermates. In contrast, the expression of the p300 1 transgene in p300 1 transgenic mice off dox lead to significant impairments in long-term recognition memory and contextual fear memory, as well as significant reduction in $\mathrm{H} 3$ acetylation in the forebrain. Our results suggest that p300 is specifically required for these types of memory. Morris water maze and contextual fear conditioning are both hippocampus-dependent tasks (Morris et al. 1982; Phillips and LeDoux 1992); however, increasing evidence suggests that different molecules are required for each type of memory (for review, see Mizuno and Giese 2005). Thus, it is not surprising that p300 may be involved in the molecular mechanisms involved in long-term contextual fear memory formation but not in mechanisms underlying spatial memory.

The transcriptional coactivator functions of p300 appear to be exerted through multiple mechanisms: p300 functions as a scaffold, forming a multiprotein transcriptional regulatory complex, and through its acetyltranferase enzymatic activity can influence histone and nonhistone proteins activity (for review, see Chan and La Thangue 2001). Similar to CBP $\Delta 1$ (Wood et al. 2005), p300 $\Delta 1$ binds to molecules that interact with the aminoterminal domain of p300. Thus, factors that bind to p300 1 will not recruit HAT activity and carboxy-terminal activation domains of p300. p300 binds to transcription factors that have been shown to be involved in memory formation (e.g., CREB and family members, c-Fos, Elk-1, C/EBP, NF-кB) (see Kasper et al. 2006). The memory impairments observed in this study may be a result of impaired CRE-dependent transcription, as we observed that p30041 inhibits CRE-dependent transcription in cell culture. However, we cannot rule out the idea that other transcription factors that interact with p300 may also be involved. Regulation of gene expression is a multifactorial and combinatorial process that involves a set of transcription factors and coactivators. Previous studies have implicated $\mathrm{CBP}$ as a critical transcriptional coactivator in long-term memory formation (Oike et al. 1999; Bourtchouladze et al. 2003; Alarcon et al. 2004; Korzus et al. 2004; Wood et al. 2005, 2006), and the present study constitutes the first evidence that p300 may also play a role in this process. Besides CREB, other transcription factors have been shown to 
A

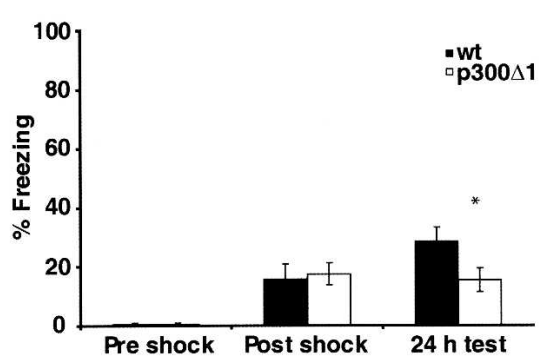

C

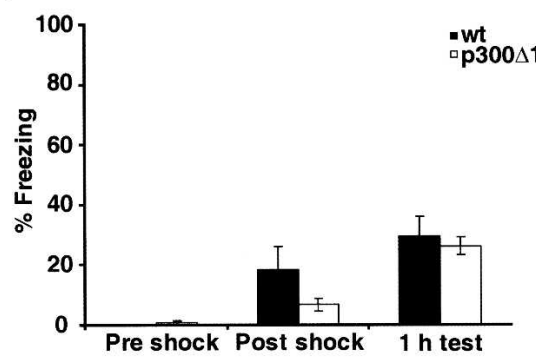

B

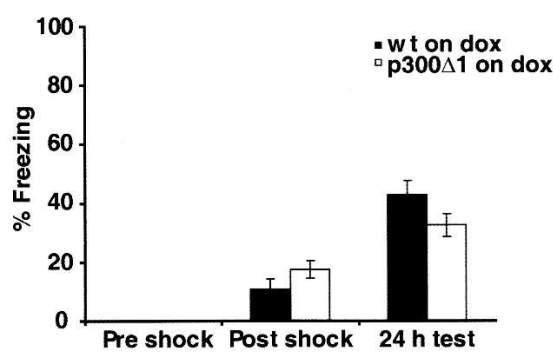

D

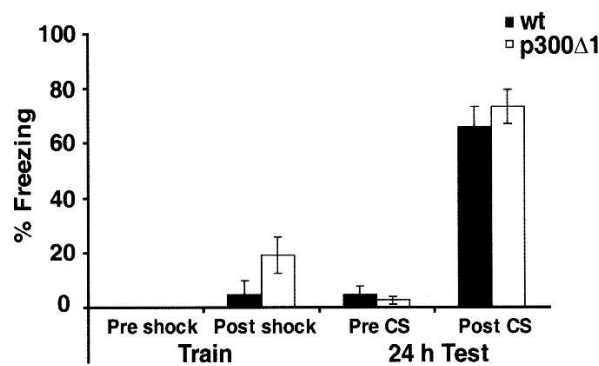

Figure 5. $p 300 \Delta 1$ transgenic mice show impaired long-term contextual fear memory. (A) p300 1 transgenic mice $(n=18)$ show significant lower percentage of freezing compared with their wild-type littermates $(n=19)$ when tested $24 \mathrm{~h}$ after training in the contextual fear conditioning. (B) p300 1 transgenic mice fed with doxycycline $(n=20)$ show a reduced (but not statistically significant) percentage of freezing compared with their wild-type littermates $(n=20)$. (C) p300 1 transgenic mice $(n=10)$ and wild-type littermates $(n=10)$ show comparable contextual fear memory tested $1 \mathrm{~h}$ after training. (D) p300 1 transgenic mice $(n=7)$ and wild-type littermates $(n=7)$ show comparable long-term cued fear memory tested $24 \mathrm{~h}$ after training.

respond to the neuronal activation triggered by learning. It is now well established that NF- $\mathrm{B}$ is required for memory formation and that NF-кB-dependent transcription requires coactivators possessing HAT activity (for review, see Quivy and Van Lint 2004; Romano et al. 2006). Zhong and colleagues showed that PKA regulates the transcriptional activity of NF- $\mathrm{kB}$ in cultured cells by modulating its interaction with CBP and p300 (Zhong et al. 1998). Also, the transcription factor Elk-1 has been implicated in associative memory formation (Cammarota et al. 2000). Interestingly, a study using fibroblasts showed that p300 is important in Elk-1 activation of transcription and that the interactions between Elk-1 and p300 change following Elk-1 phosphorylation by MAPK, resulting in increased p300 HAT activity (Li et al. 2003). Moreover, it was shown that $\mathrm{p} 300$ acetylates $\mathrm{C} / \mathrm{EBP} \beta$ at $\mathrm{K} 39$ and that this post-translational modification is required for transcription of c-fos (Cesena et al. 2007). However, regulation of NF-кB, Elk-1, and C/EBP $\beta$ activities by p300 in neuronal cells remains to be investigated.

A comparison of memory phenotypes in CBP $\Delta 1$ (Wood et al. 2005) and p300 1 transgenic mice suggests that CBP, but not p300, is required for spatial memory, revealing a differential role for these highly homologous coactivators. However, such a conclusion should be made with caution because different transgenic models may give rise to different levels and patterns of transgene expression (Furth et al. 1994), and this difference may account for the distinct behavioral phenotype. Indeed, we observed that CBP 1 transgenic mice show much lower levels of forebrain acetylation of histone $\mathrm{H} 3$ than $\mathrm{p} 300 \Delta 1$ transgenic mice (data not shown), suggesting higher levels of expression of the CBP $\Delta 1$ transgene than the p $300 \Delta 1$ transgene. On the other hand, both mice showed impaired long-term contextual fear conditioning and long-term recognition memory. Investigation of whether $\mathrm{CBP}$ and p300 are recruited interchangeably after activation trig-

gered by learning or whether they are differentially recruited by different signaling pathways that lead to transcription activation will be the subject of further studies. Increasing evidence has shown that CBP and p300 have unique functions. In vivo studies found that during embryogenesis, hematopoiesis, and motor skill learning, CBP and p300 have distinct functions (Tanaka et al. 1997; Yao et al. 1998; Kasper et al. 2002, 2006; Oliveira et al. 2006). Different substrate specificity and interaction with distinct molecules may account for these differences. McManus and Hendzel (2003) have shown that CBP has a preference for acetylating K12 on histone $\mathrm{H} 4$, whereas p300 preferentially acetylates $\mathrm{K} 8$ on histone $\mathrm{H} 4$ in vivo. Also, as mentioned above, Li et al. (2003) showed that in fibroblasts p300 is important in Elk-1 activation of transcription and that the interactions between Elk-1 and p300, but not CBP, change following Elk-1 phosphorylation by MAPK, resulting in increased p300 HAT activity. In neuronal cells, the activation of CaMKII, CaMKIV, or PKA, but not MAPK, can lead to induction of CBPmediated transcription (Hu et al. 1999). In agreement with this study, Impey et al. (2002) showed that in hippocampal neurons, CaMKIV signaling activates CBP- but not p300-dependent transcription, and that MAPK signaling is dispensable for activation of CBP (Impey et al. 2002). However, it is not known whether MAPK signaling activates p300 in neuronal cells.

Interestingly, neither CBP $\Delta 1$ (Wood et al. 2005) nor p300 1 transgenic mice showed impairments in cued fear conditioning. These findings may be explained by low levels of transgene expression in the amygdala in those mice or it may suggest that transcriptional coactivators other than CBP or p300 play a role in cued fear conditioning. In agreement with our observations, other recent studies that investigated a role for CBP in memory also found no significant deficits in cued fear conditioning in three different genetically modified CBP mutant mice (Alarcon et al. 2004; Korzus et al. 2004; Wood et al. 2006). As it is known that activation of transcription in the amygdala is essential for this form of associative memory (Bailey et al. 1999), it would be interesting to investigate whether other transcriptional coactivators with HAT activity, such as p300/CBP associated factor $(\mathrm{P} / \mathrm{CAF})$ and GCN5, play a role in this form of memory.

Transcriptional coactivators play critical roles in coordinating and integrating multiple signal-dependent events with the transcriptional apparatus allowing gene activation to occur in response to diverse stimuli. The present study suggests that the transcriptional coactivator p300 is required in the process of gene activation induced by certain forms of learning. It remains to be investigated which signaling pathways recruit p300 during memory formation. A recent study showed that some RTS patients, who have cognitive abnormalities, have mutations in the EP300 gene, some of which lead to proteins that lack the HAT domain (Roelfsema et al. 2005). Our study is the first to suggest that the impaired p300 activity may partially contribute to the cognitive impairments observed in these patients. 


\section{Materials and Methods}

\section{Immunohistochemistry}

C57Bl/6J mice were anesthetized with isoflurane and transcardially perfused with phosphate-buffered saline (PBS) followed by 4\% paraformaldehyde in PBS using a peristaltic perfusion pump. Fixed brains were dissected, post-fixed overnight, and then cryoprotected in $30 \%$ sucrose at $4^{\circ} \mathrm{C}$. Brains were flash frozen in 2-methylbutane on dry ice and mounted on cryostat chucks using OCT (Optimal Cutting Temperature compound; Sakura). Coronal sections were cut at a thickness of $30 \mu \mathrm{m}$ and collected in $1 \times$ PBS. Floating sections were washed with $1 \times$ PBS. Permeabilization was done with $0.1 \%$ Triton X-100 (Fisher), and sections were blocked in $8 \%$ normal goat serum (NGS, Vector Laboratories) with $0.3 \%$ Triton-X for $50 \mathrm{~min}$ at room temperature. Incubation with p300 antibodies (C-20 and N-15, 1:500, Santa Cruz Biotechnology) was done overnight at $4^{\circ} \mathrm{C}$ in $2 \%$ NGS, $0.3 \%$ Triton X-100 in PBS and for two hours at room temperature with goat anti-rabbit IgG-FITC secondary antibody (1:500, Santa Cruz Biotechnology). Sections were washed three times for $5 \mathrm{~min}$ each in PBS before and after each incubation step. Sections were mounted on slides in 50\% glycerol and imaged using a Leica DMRBE fluorescent microscope using a $10 \times$ objective and a Hamamatsu CCD camera.

\section{Generation of p300 1 transgenic mice}

The full-length wild-type cDNA for human p300 was kindly provided by Dr. Richard Eckner (University of New Jersey, Newark). Amino acids 1-1031 (p300s1) were cloned into pBluescript II SK $(+/-)$ using NotI and ScaI sites. The truncated p300 cDNA was then FLAG-epitope tagged by Muta-Gene Phagemid In Vitro Mutagenesis (Bio-Rad). FLAG-p300 1 was cloned into the EcoRV site of MM400, which contains the tetracycline operator sequence (tetO) and a minimal promoter (Mayford et al. 1996). All cloning junctions were verified by DNA sequencing. The FLAG-p300 1 transgene construct was excised from MM400 using NotI sites and purified by $\mathrm{CsCl}$ gradient centrifugation. Transgenic mice were generated by injecting purified TetO-FLAG-p300 $\Delta 1$ into pronuclei of B6-SJL/F1 zygotes (Transgenic and Chimeric Mouse Facility, University of Pennsylvania, Philadelphia). Founders were backcrossed to C57BL/6J mice for at least 5 generations. CaMKII $\alpha$-tTA (line B) mice were obtained from Dr. Mark Mayford (Mayford et al. 1996). Mice were maintained and bred under standard conditions, consistent with National Institutes of Health guidelines and approved by the Institutional Animal Care and Use Committee. Mice were maintained on a $12 \mathrm{~h}$ light/12 h dark cycle with the behavioral testing occurring during the light phase. For experiments subjects were 8-16 wk old at the time of testing and had free access to food and water. Littermate mice were used for controls in all experiments. For reversal experiments, animals were fed with food supplemented with $40 \mathrm{mg} / \mathrm{kg}$ doxycycline (Bio-Serv) during development and adulthood and throughout the experiment. For genotyping, tail DNA was prepared and analyzed by Southern blotting and/or PCR using transgene-specific probe and/or primers respectively (Abel et al. 1997; forward PCR primer 5'-CTTTCCCAGCCAGCTCTAAG-3'; reverse PCR primer 5'-GGAAAGTCCTTGGGGTCTTC-3').

\section{Cell culture}

HEK 293 cells (ATCC) were maintained in Minimum Essential Media with Earle's Salts (GIBCO) supplemented with $10 \%$ horse serum (ATCC) and 1\% penicillin/streptomycin (LTI) and grown at $37^{\circ} \mathrm{C}, 5 \% \mathrm{CO}_{2}$. Before transfection, cells at $60 \%-70 \%$ confluence were incubated in serum-free media with $1 \%$ penicillin/ streptomycin for $3 \mathrm{~h}$. Cells were transfected with a total of $375 \mathrm{ng}$ plasmid DNA using FuGene 6 Transfection Reagent (Roche) according to manufacturer's protocol. The following plasmids were used: pßgal-Control (BD Biosciences), CRE-Luciferase (Oh et al. 2003), and p300s1-MM400. pßgal-Control contains the SV40 early promoter and enhancer sequences inserted upstream and downstream, respectively, of the lac $Z$ gene and was used to normalize the transfection efficacy. CRE-luciferase expresses firefly luciferase under the control of a CRE and functions as a reporter of CRE-mediated transcription. p300 1 -MM400 expresses p300 1 (residues 1-1031) from the CMV promoter. MM400 was added to keep the amount of DNA in each transfection equal. One day after transfection, the cells were treated with $10 \mu \mathrm{M}$ of forskolin (Sigma) and $10 \mu \mathrm{M}$ of 3-isobutyl-1-methylxanthine (IBMX) (Sigma) and incubated for $6 \mathrm{~h}$. At the end of incubation, cells were harvested and assayed for luciferase activity with the Luciferase Assay System with Reporter Lysis Buffer (Promega) and $\beta$-Galactosidase activity with $\beta$-Gal Reporter Gene Assay (Roche).

\section{RT-PCR}

Preparation of mRNA and cDNA from amygdala, hippocampi, cortex, and cerebellum was done as described in Wood et al. (2006). Preparation of cDNA was performed with $3 \mu \mathrm{g}$ of total RNA. Two sets of primers were used for nested PCR. The primers recognize part of the p300 $\Delta 1$ transgene and the SV 40 polyA tail (set 1: forward 5'-CTTTCCCAGCCAGCTCTAAG-3'; reverse 5'GGAAAGTCCTTGGGGTCTTC-3'; set 2: forward 5'-TTCCCAGG AAGTGAAGATGG-3'; reverse 5'-AGATGGCATTTCTTCTGAG CA-3') and span one intron. Control primers amplify a 361-bp fragment of a constitutively expressed housekeeping gene, rig/ $\mathrm{S} 15$, which encodes a small ribosomal subunit protein (RETROscript kit, Ambion).

\section{AcH3 Western blotting}

Forebrain from one mouse was homogenized in hypotonic lysis buffer $(10 \mathrm{mM}$ Tris at $\mathrm{pH} 8.0,1 \mathrm{mM}$ EDTA, $2.5 \mathrm{mM}$ sodium pyrophosphate, $1 \mathrm{mM}$ sodium ortovanadate, $1 \mathrm{mM}$ PMSF, $1 \%$ protease inhibitor cocktail [Sigma]). The nuclear pellet was resuspended in 5 volumes of $0.2 \mathrm{M} \mathrm{HCl} / 10 \%$ glycerol and the acid supernatant was mixed with 10 volumes of ice cold acetone. The histone pellet was then resuspended in $9 \mathrm{M}$ Urea. The protein quantification was done using the Bradford reagent (Bio-Rad). Samples were loaded in a $15 \%$ acrylamide gel and blotted onto a nitrocellulose membrane (Bio-Rad). The membrane was blocked in Tris-buffered saline (TBS) and 5\% nonfat dry milk and probed with Actin (Chemicon) and AcH3 (Upstate) antibodies (1:5000 dilution in TBS containing 0.05\% Tween 20 [TBST] and 2\% milk). Blots were then incubated with AP-conjugated anti-rabbit or anti-mouse secondary antibodies (1:5000 dilution in TBST and $2 \%$ milk, Jackson ImmunoResearch). Blots were then incubated in enhanced chemifluorescent substrate (Amersham) and exposed to a fluorescence scanner (Storm, Amersham).

\section{Water maze}

The hidden platform experiment was performed using the method previously described (Wood et al. 2005).

\section{Fear conditioning}

The fear conditioning experiments were performed using the methods previously described (Wood et al. 2005).

\section{Novel object recognition}

The experimental apparatus consisted of a white rectangular open field $(60 \mathrm{~cm} \times 50 \mathrm{~cm} \times 26 \mathrm{~cm})$. Prior to training, mice were handled for one minute a day for two days and habituated to the experimental apparatus for five minutes in the absence of objects. During the training phase, mice were placed in the experimental apparatus in the presence of two identical objects and allowed to explore for $15 \mathrm{~min}$. After a retention interval of 30 min or $24 \mathrm{~h}$, mice were placed again in the apparatus where this time one of the objects was replaced by a novel one. Mice were allowed to explore for $15 \mathrm{~min}$. Objects were rinsed with ethanol between trials and before first trial. All testing and training sessions were videotaped and analyzed by an experimenter blind to the genotype of the animals. It was considered exploration of the objects when mice were facing the objects and/or touching it. Preference for the novel object was expressed as the percent time spent exploring the novel object relative to the total time spent exploring both objects. The identity of the objects-which one 
was novel or familiar-as well as the spatial locations where they were located were balanced between subjects.

\section{Statistical analyses}

Statistical analyses were performed using Student's $t$-test, with the exception of the Western blot analysis of acetyl histone $\mathrm{H} 3$ levels in forebrain of wild-type and transgenic mice and the Morris water maze experiments. In the Western blot experiment, two-way analyses of variance (ANOVA) were performed using Statistica 7. In the Morris water maze experiment, two-way repeated-measures ANOVA were performed using SigmaStat. To compare groups, we used the Student-Newman-Keuls multiple comparisons post-hoc test. All experiments were done by an individual blind to genotype.

\section{Acknowledgments}

We thank Dr. Jean Richa at the Transgenic and Chimeric Mouse Facility at the University of Pennsylvania for generating tetOp300 1 transgenic animals, Dr. Mark Mayford for the CaMKII $\alpha$-tTA line B mice, and Dr. Richard Eckner for providing us p300 cDNA. We thank Christopher Vecsey for comments on the manuscript. This research was supported by a predoctoral fellowship from Fundação para a Ciência e Tecnologia, Portugal (A.M.M.O.), a SFN MNFP postdoctoral fellowship (M.A.W.), and an Alavi-Dabiri postdoctoral fellowship (M.A.W.), and by grants from the National Institutes of Health, the Human Frontiers Science Program, and the Packard Foundation (T.A.).

\section{References}

Abel, T., Nguyen, P.V., Barad, M., Deuel, T.A., Kandel, E.R., and Bourtchouladze, R. 1997. Genetic demonstration of a role for PKA in the late phase of LTP and in hippocampus-based long-term memory. Cell 88: 615-626.

Alarcon, J.M., Malleret, G., Touzani, K., Vronskaya, S., Ishii, S., Kandel, E.R., and Barco, A. 2004. Chromatin acetylation, memory, and LTP are impaired in $\mathrm{CBP}^{+/-}$mice: A model for the cognitive deficit in Rubinstein-Taybi syndrome and its amelioration. Neuron 42: 947-959.

Bailey, D.J., Kim, J.J., Sun, W., Thompson, R.F., and Helmstetter, F.J. 1999. Acquisition of fear conditioning in rats requires the synthesis of mRNA in the amygdala. Behav. Neurosci. 113: 276-282.

Bartholdi, D., Roelfsema, J.H., Papadia, F., Breuning, M.H., Niedrist, D., Hennekam, R.C., Schinzel, A., and Peters, D.J. 2007. Genetic heterogeneity in Rubinstein-Taybi Syndrome: Delineation of the phenotype of the first patients carrying mutations in EP300. J. Med. Genet. 44: 327-333.

Bourtchouladze, R., Lidge, R., Catapano, R., Stanley, J., Gossweiler, S., Romashko, D., Scott, R., and Tully, T. 2003. A mouse model of Rubinstein-Taybi syndrome: Defective long-term memory is ameliorated by inhibitors of phosphodiesterase 4. Proc. Natl. Acad. Sci. 100: 10518-10522.

Burgin, K.E., Waxham, M.N., Rickling, S., Westgate, S.A., Mobley, W.C., and Kelly, P.T. 1990. In situ hybridization histochemistry of $\mathrm{Ca}^{2+} /$ calmodulin-dependent protein kinase in developing rat brain J. Neurosci. 10: 1788-1798.

Cammarota, M., Bevilaqua, L.R., Ardenghi, P., Paratcha, G., Levi de Stein, M., Izquierdo, I., and Medina, J.H. 2000. Learning-associated activation of nuclear MAPK, CREB and Elk-1, along with Fos production, in the rat hippocampus after a one-trial avoidance learning: Abolition by NMDA receptor blockade. Brain Res. Mol. Brain Res. 76: 36-46.

Cesena, T.I., Cardinaux, J.R., Kwok, R., and Schwartz, J. 2007. CCAAT/enhancer-binding protein (C/EBP) $\beta$ is acetylated at multiple lysines: Acetylation of $\mathrm{C} / \mathrm{EBP} \beta$ at lysine 39 modulates its ability to activate transcription. J. Biol. Chem. 282: 956-967.

Chan, H.M. and La Thangue, N.B. 2001. p300/CBP proteins: HATs for transcriptional bridges and scaffolds. J. Cell Sci. 114: 2363-2373.

Chrivia, J.C., Kwok, R.P., Lamb, N., Hagiwara, M., Montminy, M.R., and Goodman, R.H. 1993. Phosphorylated CREB binds specifically to the nuclear protein CBP. Nature 365: 855-859.

Eckner, R., Ewen, M.E., Newsome, D., Gerdes, M., DeCaprio, J.A., Lawrence, J.B., and Livingston, D.M. 1994. Molecular cloning and functional analysis of the adenovirus E1A-associated 300-kD protein (p300) reveals a protein with properties of a transcriptional adaptor. Genes \& Dev. 8: 869-884.

Furia, B., Deng, L., Wu, K., Baylor, S., Kehn, K., Li, H., Donnelly, R., Coleman, T., and Kashanchi, F. 2002. Enhancement of nuclear
factor-кB acetylation by coactivator $\mathrm{p} 300$ and HIV-1 Tat proteins. $J$. Biol. Chem. 277: 4973-4980.

Furth, P.A., St Onge, L., Boger, H., Gruss, P., Gossen, M., Kistner, A., Bujard, H., and Hennighausen, L. 1994. Temporal control of gene expression in transgenic mice by a tetracycline-responsive promoter. Proc. Natl. Acad. Sci. 91: 9302-9306.

Grunstein, M. 1997. Histone acetylation in chromatin structure and transcription. Nature 389: 349-352.

$\mathrm{Gu}$, W. and Roeder, R.G. 1997. Activation of p53 sequence-specific DNA binding by acetylation of the p53 C-terminal domain. Cell 90: $595-606$.

Hanson, P.I. and Schulman, H. 1992. Neuronal $\mathrm{Ca}^{2+} / \mathrm{calmodulin}^{-}$ dependent protein kinases. Annu. Rev. Biochem. 61: 559-601.

Hu, S.C., Chrivia, J., and Ghosh, A. 1999. Regulation of CBP-mediated transcription by neuronal calcium signaling. Neuron 22: 799-808.

Impey, S., Fong, A.L., Wang, Y., Cardinaux, J.R., Fass, D.M., Obrietan, K., Wayman, G.A., Storm, D.R., Soderling, T.R., and Goodman, R.H. 2002. Phosphorylation of CBP mediates transcriptional activation by neural activity and CaM kinase IV. Neuron 34: 235-244.

Kalkhoven, E. 2004. CBP and p300: HATs for different occasions. Biochem. Pharmacol. 68: 1145-1155.

Kasper, L.H., Boussouar, F., Ney, P.A., Jackson, C.W., Rehg, J., van Deursen, J.M., and Brindle, P.K. 2002. A transcription-factor-binding surface of coactivator p300 is required for haematopoiesis. Nature 419: 738-743.

Kasper, L.H., Fukuyama, T., Biesen, M.A., Boussouar, F., Tong, C., de Pauw, A., Murray, P.J., van Deursen, J.M., and Brindle, P.K. 2006. Conditional knockout mice reveal distinct functions for the global transcriptional coactivators CBP and p300 in T-cell development. Mol. Cell. Biol. 26: 789-809.

Korzus, E. 2003. The relation of transcription to memory formation. Acta Biochim. Pol. 50: 775-782.

Korzus, E., Rosenfeld, M.G., and Mayford, M. 2004. CBP histone acetyltransferase activity is a critical component of memory consolidation. Neuron 42: 961-972.

Levenson, J.M., O'Riordan, K.J., Brown, K.D., Trinh, M.A., Molfese, D.L., and Sweatt, J.D. 2004. Regulation of histone acetylation during memory formation in the hippocampus. J. Biol. Chem. 279: 40545-40559.

Li, Q., Xiao, H., and Isobe, K. 2002. Histone acetyltransferase activities of cAMP-regulated enhancer-binding protein and p300 in tissues of fetal, young, and old mice. J. Gerontol. A Biol. Sci. Med. Sci. 57: B93-B98.

Li, Q.J., Yang, S.H., Maeda, Y., Sladek, F.M., Sharrocks, A.D., and Martins-Green, M. 2003. MAP kinase phosphorylation-dependent activation of Elk-1 leads to activation of the co-activator p300. EMBO J. 22: 281-291.

Lundblad, J.R., Kwok, R.P., Laurance, M.E., Harter, M.L., and Goodman, R.H. 1995. Adenoviral E1A-associated protein p300 as a functional homologue of the transcriptional co-activator CBP. Nature 374: $85-88$.

Maren, S. and Quirk, G.J. 2004. Neuronal signalling of fear memory. Nature Rev. Neurosci. 5: 844-852.

Mayford, M., Bach, M.E., Huang, Y.Y., Wang, L., Hawkins, R.D., and Kandel, E.R. 1996. Control of memory formation through regulated expression of a CaMKII transgene. Science 274: 1678-1683.

McManus, K.J. and Hendzel, M.J. 2003. Quantitative analysis of CBPand P300-induced histone acetylations in vivo using native chromatin. Mol. Cell. Biol. 23: 7611-7627.

Mizuno, K. and Giese, K.P. 2005. Hippocampus-dependent memory formation: Do memory type-specific mechanisms exist? J. Pharmacol. Sci. 98: 191-197.

Morris, R.G., Garrud, P., Rawlins, J.N., and O'Keefe, J. 1982. Place navigation impaired in rats with hippocampal lesions. Nature 297: 681-683.

Oh, D.Y., Wang, L., Ahn, R.S., Park, J.Y., Seong, J.Y., and Kwon, H.B. 2003. Differential G protein coupling preference of mammalian and nonmammalian gonadotropin-releasing hormone receptors. Mol. Cell. Endocrinol. 205: 89-98.

Oike, Y., Hata, A., Mamiya, T., Kaname, T., Noda, Y., Suzuki, M., Yasue, H., Nabeshima, T., Araki, K., and Yamamura, K. 1999. Truncated CBP protein leads to classical Rubinstein-Taybi syndrome phenotypes in mice: Implications for a dominant-negative mechanism. Hum. Mol. Genet. 8: 387-396.

Oliveira, A.M., Abel, T., Brindle, P.K., and Wood, M.A. 2006. Differential role for CBP and p300 CREB-binding domain in motor skill learning. Behav. Neurosci. 120: 724-729.

Phillips, R.G. and LeDoux, J.E. 1992. Differential contribution of amygdala and hippocampus to cued and contextual fear conditioning. Behav. Neurosci. 106: 274-285.

Quivy, V. and Van Lint, C. 2004. Regulation at multiple levels of NF-кB-mediated transactivation by protein acetylation. Biochem. 
Pharmacol. 68: 1221-1229.

Roelfsema, J.H., White, S.J., Ariyurek, Y., Bartholdi, D., Niedrist, D., Papadia, F., Bacino, C.A., den Dunnen, J.T., van Ommen, G.J., Breuning, M.H., et al. 2005. Genetic heterogeneity in Rubinstein-Taybi syndrome: Mutations in both the CBP and EP300 genes cause disease. Am. J. Hum. Genet. 76: 572-580.

Romano, A., Freudenthal, R., Merlo, E., and Routtenberg, A. 2006. Evolutionarily-conserved role of the NF-kB transcription factor in neural plasticity and memory. Eur. J. Neurosci. 24: 1507-1516.

Tanaka, Y., Naruse, I., Maekawa, T., Masuya, H., Shiroishi, T., and Ishii, S. 1997. Abnormal skeletal patterning in embryos lacking a single Cbp allele: A partial similarity with Rubinstein-Taybi syndrome. Proc. Natl. Acad. Sci. 94: 10215-10220.

Tremblay, P., Meiner, Z., Galou, M., Heinrich, C., Petromilli, C., Lisse, T., Cayetano, J., Torchia, M., Mobley, W., Bujard, H., et al. 1998. Doxycycline control of prion protein transgene expression modulates prion disease in mice. Proc. Natl. Acad. Sci. 95: $12580-12585$.

Vo, N. and Goodman, R.H. 2001. CREB-binding protein and p300 in transcriptional regulation. J. Biol. Chem. 276: 13505-13508.

Wood, M.A., Kaplan, M.P., Park, A., Blanchard, E.J., Oliveira, A.M., Lombardi, T.L., and Abel, T. 2005. Transgenic mice expressing a truncated form of CREB-binding protein (CBP) exhibit deficits in hippocampal synaptic plasticity and memory storage. Learn. Mem.
12: $111-119$.

Wood, M.A., Attner, M.A., Oliveira, A.M., Brindle, P.K., and Abel, T. 2006. A transcription factor-binding domain of the coactivator CBP is essential for long-term memory and the expression of specific target genes. Learn. Mem. 13: 609-617.

Yao, T.P., Oh, S.P., Fuchs, M., Zhou, N.D., Ch'ng, L.E., Newsome, D., Bronson, R.T., Li, E., Livingston, D.M., and Eckner, R. 1998. Gene dosage-dependent embryonic development and proliferation defects in mice lacking the transcriptional integrator p300. Cell 93: $361-372$.

Yuan, L.W. and Gambee, J.E. 2001. Histone acetylation by p300 is involved in CREB-mediated transcription on chromatin. Biochim. Biophys. Acta 1541: 161-169.

Zhong, H., Voll, R.E., and Ghosh, S. 1998. Phosphorylation of NF-кB p65 by PKA stimulates transcriptional activity by promoting a novel bivalent interaction with the coactivator $\mathrm{CBP} / \mathrm{p} 300$. Mol. Cell 1: 661-671.

Zou, D.J., Greer, C.A., and Firestein, S. 2002. Expression pattern of $\alpha$ CaMKII in the mouse main olfactory bulb. J. Comp. Neurol. 443: $226-236$.

Received May 30, 2007; accepted in revised form July 9, 2007. 


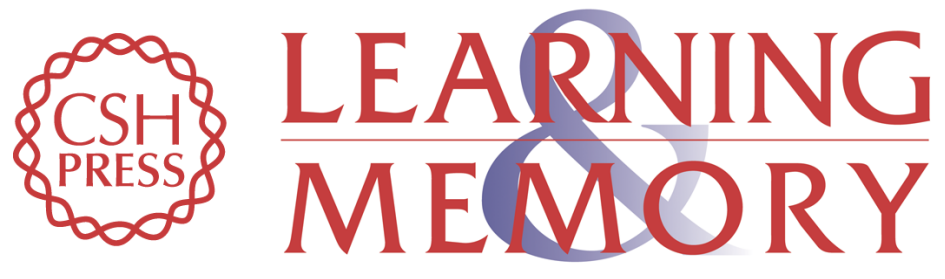

\section{Transgenic mice expressing an inhibitory truncated form of p300 exhibit long-term memory deficits}

Ana M.M. Oliveira, Marcelo A. Wood, Conor B. McDonough, et al.

Learn. Mem. 2007, 14:

Access the most recent version at doi:10.1101//m.656907

References This article cites 48 articles, 18 of which can be accessed free at:

http://learnmem.cshlp.org/content/14/9/564.full.html\#ref-list-1

License

Email Alerting Receive free email alerts when new articles cite this article - sign up in the box at the Service top right corner of the article or click here. 